\title{
A Nomogram to Predict Postoperative Complications in Elderly with Total Hip Replacement
}

\section{Xiujuan Tan ( $\nabla 307319679 @ q q . c o m)$}

The First Affiliated Hospital, Jinan University https://orcid.org/0000-0003-0608-9045

\section{Fengmin Ge}

The Affiliated Hospital of Guangdong Medical University

\section{Guixi Mo}

The Affiliated Hospital of Guangdong Medical University

\section{Zhiyi Li}

The Affiliated Hospital of Guangdong Medical University

\section{Xiaoxia Gu}

The Affiliated Hospital of Guangdong Medical University

\section{Liangqing Zhang}

The Affiliated Hospital of Guangdong Medical University

\section{Research article}

Keywords: elderly, total hip replacement, postoperative complication, nomogram

Posted Date: October 6th, 2020

DOI: https://doi.org/10.21203/rs.3.rs-79448/v1

License: (c) (i) This work is licensed under a Creative Commons Attribution 4.0 International License. Read Full License

Version of Record: A version of this preprint was published at World Journal of Clinical Cases on April 26th, 2022. See the published version at https://doi.org/10.12998/wjcc.v10.i12.3720. 


\section{Abstract}

Background: By analyzing the risk factors of postoperative complications in elderly patients with hip replacement, We aimed to develop a nomogram model based on preoperative and intraoperative variables and verified the sensitivity and specificity for risk stratification of postoperative complications in elderly with total hip replacement patients.

Methods: A total of 414 elderly patients who underwent surgical treatment for total hip replacement hospitalized at the Affiliated Hospital of Guangdong Medical University from March 1, 2017 to August 31, 2019 were included into this study. Univariate and multivariate logistic regression were conducted to identify independent risk factors of postoperative complication in the 414 patients. A nomogram was developed by $\mathrm{R}$ software and validated to predict the risk of postoperative complications.

Results: Multivariate logistic regression analysis revealed that age $(\mathrm{OR}=1.05,95 \% \mathrm{Cl}: 1.00-1.09)$, renal failure(OR=0.90, 95\% Cl: 0.83ه0.97) , Type2 diabetes (OR=1.05, 95\% Cl: $1.00 \otimes 1.09)$, ALB (OR=0.91, 95\% Cl: $0.83 \varangle 0.99$ ) were independent risk factors of postoperative complication in elderly patients with hip replacement (Pख0.05) . For validation of the nomogram, ROC curve revealed that the model predicting postoperative complication in elderly patients with hip replacement was the area under the curve of $0.8254(95 \% \mathrm{Cl}: 0.78 \otimes 0.87)$, the slope of the calibration plot was close to 1 and the model passed HosmerLemeshow goodness of fit test $\left(x^{2}=10.16, P=0.4264\right)$, calibration in $R$ Emax $=0.176$, Eavg $=0.027$, which all demonstrated that the model was of good accuracy.

Conclusion: The nomogram predicting postoperative complications in patients with total hip replacement constructed based on age, type 2 diabetes, renal failure and ALB is of good discrimination and accuracy, which was of clinical significance.

\section{Background}

Hip replacement is a frequently done and highly successful surgical intervention [1]. More than one million hip arthroplasties are performed annually worldwide [2]. Kurtz and colleagues [3] projected the demand for primary THA to grow $174 \%$ to 572,000 procedures per year by 2030 . These procedures carry a complication rate estimated to be between $2 \%$ and $14 \%$; higher complication rates are associated with more elderly and comorbid patient populations [4]. Numerous clinical tools have been developed to predict a variety of THA patient outcomes [5], but fewer to predict complications after surgery and no one is modelling by Chinese. As an anesthetist, when we go to the inpatient ward for preoperative evaluation, patient often ask: what is the proportion of risk in my surgery[The answer is perhaps or we don't know. So we want to create a tool not only can predict the risk precisely, but also guide the clinical work. Therefore this study will analyze clinical data, explored the independent risk factors for postoperative complications in elderly patients undergoing total hip replacement, develop a nomogram for accurate risk stratification of postoperative complications based on preoperative and intraoperative variables, and verify whether this tool would have good predictive for patients undergoing total hip replacement in our hospital. 


\section{Methods \\ Patients}

Approved by the Ethics Committee of the Affiliated Hospital of Guangdong Medical University committee (PJ2020-022), we were collected from all patients undergoing total hip replacement from March 1, 2017 to August 31, 2019 at the Affiliated Hospital of Guangdong Medical University. Inclusion criteria were age > $65 y$, elective surgical treatment with total hip replacement. Non-inclusion criteria were age $<65$ y, electronic medical records incomplete, patients or family members disagree with the study.

\section{Research Methods}

Data on general patient information, comorbidities, laboratory test results, intraoperative variables, and postoperative complications during hospitalization were collected from electronic medical records and electronic Anesthesia Information Management System. Known patient-related factors of complications are age, gender, fractures or not and comorbidity such as renal failure $\square$ hypertension $\square$ diabetes $\llbracket$ coronary heart disease $\llbracket$ stroke. Laboratory test results of white blood cell (WBC), red blood cell (RBC), hemoglobin $(\mathrm{HB})$, platelets (PLT), albumin (ALB) and blood urea nitrogen (BUN). Intraoperative variables included estimated blood loss (EBL), lowest heart rate, the type of anesthesia, preoperative ASA score (American Society of Anesthesiologists Score) and operation time.

The primary outcome measure was the incidence of a postoperative complication or death during hospitalization. Complications were identified from diagnoses in discharge summaries, operative reports, and ICD-9 codes by a single investigator. Included: cardiac arrest, deep venous thrombosis, myocardial infarction, pneumonia, pulmonary embolism, systemic inflammatory response syndrome, infection, dislocation, delirium, according to definitions from the NSQIP [6].

\section{Statistical analysis}

All statistical analyses were performed using the STATA14.0 statistical software package and R software (R3.2.3) with rms package added. Differences between patients with and without complications were compared with t-test or Wilcoxon rank-sum test using the mean \pm SD and the median (range) for quantitative variables and chi-squared test using $n(\%)$ for qualitative variables. Univariate logistic regression for patients clinical data were analyzed to identify the independent risk factors for postoperative complications after surgery. A multivariate logistic regression model was built using the factors associated with $p$ values $<0.05$ by univariate analysis. Selection values of $p<0.05$ variables by multivariate analysis to create a nomogram model by R software (R3.2.3) rms package, evaluated the nomogram by assessing discrimination and calibration.

\section{Results}


In our study 414 elderly total hip replacement patients of 59 cases with postoperative complications after surgery, the incidence was $14.3 \%$. The demographics and descriptive statistics for our patient cohort are given in Table 1. Multivariable logistic regression of each clinical variable of patients related factors for postoperative complications include patients age $(\mathrm{OR}=1.05,95 \% \mathrm{Cl}: 1.00 \sim 1.09)$, renal failure $(\mathrm{OR}=0.90$, $95 \% \mathrm{Cl}: 0.83 \sim 0.97)$, diabetes(OR $=2.37,95 \% \mathrm{Cl}: 1.04 \sim 5.40)$ and $\mathrm{ALB}(\mathrm{OR}=0.91,95 \% \mathrm{Cl}: 0.83 \sim 0.99)$ (Table 2).

According to the above multivariable logistic regression analysis results, choose $p<0.05$ variable apply $R$ Software to build a nomogram model of postoperative complications in elderly total hip replacement patients (Fig. 1).

Using nomogram model to predict postoperative complications in elderly total hip replacement patients risk ROC curve, AUC is $0.8254(95 \% \mathrm{Cl}: 0.78 \otimes 0.87$ ), indicating that the nomogram model has a good bootstrap-corrected concordance(Fig. 2).

Perform Hosmer-Lemeshow goodness-of-fit test and evaluate nomogram Model accuracy, HosmerLemeshow fit goodness test $x^{2}=10.16, P=0.4264$ (Fig. 3), calibration in rms package by $R$ software Emax $=0.176$, Eavg $=0.027$ (Fig. 4), all shows that the model appears to be well-calibrated, with predicted outcome rates closely reflecting the observed rates. 
Table 1

Comparison of clinical data between No Complications and Complications.

\begin{tabular}{|c|c|c|c|c|}
\hline Patient Characteristics & $\begin{array}{l}\text { No Complications } \\
(\mathrm{N}=355)\end{array}$ & $\begin{array}{l}\text { Complications } \\
(\mathrm{N}=59)\end{array}$ & $t / x^{2} / Z$ Value & P Value \\
\hline $\operatorname{Age}(y)^{a}$ & $74.06 \pm 7.25$ & $81.29 \pm 8.19$ & 6.96 & 0.000 \\
\hline Male gender (\%) ${ }^{b}$ & 149(41.9) & $24(40.7)$ & 0.03 & 0.852 \\
\hline Weight(kg) ${ }^{a}$ & $58.93 \pm 10.23$ & $57.2 \pm 9.93$ & 1.2 & 0.226 \\
\hline \multicolumn{5}{|l|}{ Comorbidities } \\
\hline Hypertension (\%) ${ }^{b}$ & $114(32.1)$ & $30(50.8)$ & 7.83 & 0.006 \\
\hline Diabetes $(\%){ }^{b}$ & $50(14.1)$ & $16(27.1)$ & 6.41 & 0.017 \\
\hline Coronary heart disease (\%) ${ }^{b}$ & $30(5)$ & $10(16.9)$ & 4.19 & 0.041 \\
\hline Stroke (\%) ${ }^{\mathrm{b}}$ & $16(4.5)$ & $12(20.3)$ & 20.11 & 0.000 \\
\hline Renal failure (\%) $\mathrm{b}$ & 13(3.7) & $7(11.9)$ & 7.404 & 0.017 \\
\hline Fractures (\%) ${ }^{b}$ & $123((34.6)$ & $45(76.3)$ & 36.35 & 0.000 \\
\hline \multicolumn{5}{|l|}{ Laboratory data } \\
\hline $\operatorname{WBC}\left(10^{9} / \mathrm{L}\right)^{a}$ & $7.6 \pm 2.46$ & $8.69 \pm 2.93$ & 2.7 & 0.003 \\
\hline $\operatorname{RBC}\left(10^{12} / \mathrm{L}\right)^{a}$ & $4.21 \pm 0.59$ & $3.89 \pm 0.69$ & 3.81 & 0.000 \\
\hline $\mathrm{HB}(\mathrm{g} / \mathrm{L})^{a}$ & $123.37 \pm 20.99$ & $111.89 \pm 22.64$ & 3.85 & 0.000 \\
\hline $\operatorname{PLT}\left(10^{9} / \mathrm{L}\right)^{a}$ & $235.66 \pm 71.56$ & $247.91 \pm 115.27$ & 0.79 & 0.272 \\
\hline $\operatorname{ALB}(g / L)^{a}$ & $39.37 \pm 4.51$ & $35.75 \pm 4.41$ & 5.72 & 0.000 \\
\hline $\mathrm{BUN}(\mathrm{mmol} / \mathrm{L})^{a}$ & $6.05 \pm 4.02$ & $7.71 \pm 5.08$ & 2.39 & 0.019 \\
\hline \multicolumn{5}{|l|}{ Intraoperative characteristics } \\
\hline $\mathrm{EBL}(\mathrm{ml})^{\mathrm{c}}$ & $203.79(50-1500)$ & $229.8(50-1000)$ & 1.5 & 0.134 \\
\hline Lowest heart rate(bpm $)^{a}$ & $59.39 \pm 10.49$ & $65.37 \pm 13.88$ & 3.16 & 0.001 \\
\hline Operation time $(\min )^{a}$ & $100.85 \pm 40.26$ & $89.41 \pm 37.24$ & 2.04 & 0.505 \\
\hline General Anesthesia (\%) ${ }^{b}$ & $159(44.8)$ & $24(40.7)$ & 0.35 & 0.556 \\
\hline ASA Class $\otimes$ or $\otimes(\%){ }^{b}$ & $159(44.8)$ & $518(86.4)$ & 39.35 & 0.000 \\
\hline
\end{tabular}


Preoperative patient characteristics for 414 total hip replacement procedures between March 1, 2017 to August 31,2019 . Results are presented as number (percentage) of patients, or as mean \pm standard deviation. The $\mathrm{P}$ values were obtained from chi-square tests, $\mathrm{t}$-tests, or Wilcoxon rank-sum tests, as indicated (a t-test; b chi-square test; c Wilcoxon rank-sum test).

Table 2

Multivariable logistic regression analysis results

\begin{tabular}{|llllll|}
\hline Variables & OR & SE & Z & $95 \% \mathrm{Cl}$ & P Value \\
\hline Age & 1.05 & 0.02 & 2.04 & $1.00 \sim 1.09$ & 0.041 \\
\hline Renal failure & 3.96 & 2.44 & 2.23 & $1.18 \sim 13.27$ & 0.026 \\
\hline Hypertension & 1.31 & 0.47 & 0.74 & $0.65 \sim 2.64$ & 0.457 \\
\hline Coronary heart disease & 0.84 & 0.41 & 0.36 & $0.32 \sim 2.18$ & 0.722 \\
\hline Diabetes & 2.37 & 1.00 & 2.05 & $1.04 \sim 5.40$ & 0.040 \\
\hline Stroke & 2.41 & 1.15 & 1.84 & $0.94 \sim 6.16$ & 0.066 \\
\hline Fractures & 2.21 & 0.95 & 1.86 & $0.96 \sim 5.12$ & 0.063 \\
\hline ASA Class $₫$ or $\otimes$ & 1.85 & 0.68 & 1.69 & $0.91 \sim 3.79$ & 0.090 \\
\hline Lowest heart rate & 1.02 & 0.01 & 1.10 & $0.99 \sim 1.05$ & 0.272 \\
\hline RBC & 1.05 & 0.34 & 0.16 & $0.56 \sim 1.99$ & 0.869 \\
\hline WBC & 1.03 & 0.64 & 0.44 & $0.91 \sim 1.16$ & 0.657 \\
HB & 1.00 & 0.01 & 0.10 & $0.98 \sim 1.02$ & 0.922 \\
\hline ALB & 0.90 & 0.04 & 2.73 & $0.83 \sim 0.97$ & 0.006 \\
\hline BUN & 1.00 & 0.04 & 0.13 & $0.94 \sim 1.08$ & 0.893 \\
\hline
\end{tabular}

\section{Discussion}

China is the most populated country in the world, and now has the second-largest economy in the world [7]. As nearly 166 million Chinese are aged more than 65 years [8]. The demand for healthcare, including hip arthroplasty is increasing $[9,10]$. There are several predict complications model have be reported [11, $12,13]$, but on one is modeling by Chinese. In this study, 59 of the 414 elderly total hip replacement patients who underwent surgical treatment were developed postoperative complication, the incidence is $14.3 \%$, morbidity is much higher than $3.9 \%$ [14]. Probably because our definition of complications not only included dislocation, pulmonary embolism, and infection as reported previously, but included systemic inflammatory response syndrome, delirium which are common in elderly. Among 566 older patients (mean age, 76.7 years) undergoing a variety of elective operations (including orthopedic, general, and vascular), $23.9 \%$ patients developed postoperative delirium [15]. The incidence of postoperative delirium 
was reported as $7.0 \%-30.2 \%$ in hip arthroplasty $[16,17]$. In this study the average age is $75.09 \pm 7.8$. As a result, the morbidity $14.3 \%$ is considered to be reasonable.

Age is a recognized risk factor for postoperative complications. The results of this study indicate that elderly patients with renal failure and diabetes are more likely to have postoperative complications after total hip replacement. It is Consistent with the research results of Robert K [18] et al. One possible reason is that elderly patients have more comorbidities will make them less able to withstand the stresses of anesthesia and surgery $[19,20]$. Diabetes have been reported to be significant predictors for complications such as surgical site infections [21]. The available data suggest that diabetes may promote the development of osteoarthritis [22]. Our results for the outcome measure indicate that elderly patients with renal failure and diabetes increase the weight of the nomogram model score by 25 points and 24 points, respectively.

Interestingly, our data suggest that low preoperative albumin levels can predict the incidence of postoperative complications following surgery for total hip replacement. Since ALB is a biomarker of visceral protein and immune-competence status, it is commonly used for nutritional assessment [23]. Preoperative albumin bears strong potential as a practical metric to assess a patient's overall health [24]. Recent studies even show that low ALB rather reflects a state of persistent inflammation [25]. Our results pointed out that the weight of 12.7 points in the nomogram model score will be increased for every $5 \mathrm{~g} / \mathrm{L}$ decrease in ALB. We recommend surgeons and anesthetists should ideally attempt to optimize patient nutritional status before total hip replacement in elderly in order to avoid a greater likelihood of postoperative complications or mortality.

However our study has several limitations. First, our data were limited only 414 patients, it only represents an elective patient population. Second, complications were only collected while in hospital, some of these complications could have occurred after discharge. Third, the predict model quality checks only with internal validation, so external validation will have to be included in future studies in order to promote use. Fourth, this was a retrospective study that relied on 9th edition coding, which can lead to errors and/or incomplete coding.

Risk calculators should serve as a tool to help clinical decision-making, promote individualized medicine, and aid in the shared decision-making process [26]. Many of the studies report poor discrimination and calibration of the investigated risk calculators. In our study, founded that age, diabetes, renal failure, and Albumin value are independent risk factors for postoperative complications in elderly patients with total hip replacement, ROC curve shows the AUC is 0.8254 , indicating that the nomogram model has a good discrimination. The Hosmer-Lemeshow fit goodness test $x^{2}=10.16, P=0.4264$ and calibration curve is a straight line with a slope close to 1 , indicating that the nomogram model has good accuracy in predicting the risk of postoperative complications in elderly patients with total hip replacements surgery and has clinical application value.

\section{Conclusions}


This study created a nomogram model based on age, diabetes, renal failure, and Albumin value independent risk factors for postoperative complications, has g good indexing and accuracy can provide scientific guidance for individualized clinical prevention and treatment of postoperative complications in elderly patients with total hip replacements surgery in our hospital. This four variables are easy to get in clinical practice, has clinical application value especially for Basic-level hospital.

\section{Abbreviations}

ASA: American Society of Anesthesiologists; THA: Total hip arthroplasty; NSQIP: National Surgical Quality Improvement Program; SD: Standard error; ROC: Receiver operating characteristic curve; AUC: Area under curve

\section{Declarations}

\section{Ethics approval and consent to participate}

This study was approved by the ethics committee of the Affiliated Hospital of Guangdong Medical University with the reference number PJ2020-022. All participants were informed and asked for written informed consent.

\section{Consent for publication}

Not applicable.

\section{Availability of data and materials}

The datasets generated and/or analyzed during the current study will be available from the corresponding author on reasonable request.

\section{Competing interests}

The authors declare that they have no competing interests.

\section{Funding}

No-funding. XJ T and XX G are designer of the study, collecting data, statistical analysis, interpretation of results and writing the manuscript together.

\section{Authors' contributions}

XJ T is first Author, participated in protocol writing, collecting data, statistical analysis, interpretation of results and manuscript writing. FM G helped collection of cases. ZY L participated in protocol writing, essay writing. XX G helped interpretation of results and manuscript writing. GX M and LQ Z did the 
statistical analysis and reviewed the manuscript. All authors have read and approved the final submitted manuscript.

\section{Acknowledgements}

We thank Dr. JY W of the Affiliated Hospital of Guangdong Medical University Department of Scientific Research for his excellent assistance in the data analysis.

\section{Authors' information}

$\mathrm{XX} \mathrm{G}$ and $\mathrm{LQ} \mathrm{Z}$ is Co-Corresponding author.

1, Department of Anesthesiology, the First Affiliated Hospital, Jinan University, No.601 West Huangpu Avenue, Tianhe District Guangzhou City 510632, Guangdong Province, China.

2, Department of Anesthesiology, the Affiliated Hospital of Guangdong Medical University, No.57 South People's Avenue, Xiashan District, Zhanjiang City 524001, Guangdong Province, China.

\section{References}

1. Ferguson RJ, Palmer AJ, Taylor A, et al. Hip replacement Lancet. 2018;392(10158):1662-71.

2. Pivec R, Johnson AJ, Mears SC, Mont MA. Hip arthroplasty. Lancet. 2012;380:1768-77.

3. Kurtz S, Ong K, Lau E, et al. Projections of primary and revision hip and knee arthroplasty in the United States from 2005 to 2030. J Bone Joint Surg Am. 2007;89:780-5.

4. Nanjayan SK, Swamy GN, Yellu S, Yallappa S, Abuzakuk T, Straw R. In-hospital complications following primary total hip and knee arthroplasty in octogenarian and nonagenarian patients. $J$ Orthop Traumatol. 2014;15(1):29-33.

5. Joseph F, Konopka MD. MS, et al. Risk Assessment Tools Used to Predict Outcomes of Total Hip and T otal Knee Arthroplasty. Orthop Clin N Am. 2015;46:351-62.

6. Khuri SF, Daley J, Henderson W, et al. The National Veterans Administration Surgical Risk Study: risk adjustment for the comparative assessment of the quality of surgical care. J Am Coll Surg. 1995;180:519.

7. No authors listed. Open Knowledge Repository (OKR). World Bank Group. World Development Indicators 2017. https://openknowledge.worldbank.org/han-dle/10986/26447 (date last accessed 8 July 2019).

8. No authors listed. National Bureau of Statistics of China. China Statistics Press. China Statistical Yearbook 2017. http://www.stats.gov.cn/tjsj/ndsj/2019/indexeh.htm).

9. Wang K. Brief discussion on present status and future of joint replacement in China. Chin J Joint Surg (Electronic Edition). 2015;9:12-4. http://www.cnki.com.cn/Article/CJFDTotalZHGJ201506004.htm. (date last accessed 8 August 2019) (In Chinese). 
10. Pei FX. The current status and future perspective of hip and knee arthroplasty in China. Chinese Journal of Bone Joint. 2012;1:4-8. http://www.cnki.com.cn/Article/CJFDTotalGZGL201201019.htm. (date last accessed 8 August 2019) (In Chinese).

11. Wuerz TH, Kent DM, Malchau H, et al. A nomogram to predict major complications after hip and knee arthroplasty. J Arthroplasty. 2014;29:1457-62.

12. Wuerz TH, Regenbogen SE, Ehrenfeld JM, et al. The Surgical Apgar Score in hip and knee arthroplasty. Clin Orthop Relat Res. 2011;469:1119-26.

13. Inneh IA, Lewis CG, Schutzer SF. Focused risk analysis: regression model based on 5,314 total hip andknee arthroplasty patients from a single institution. J Arthroplasty. 2014;29(10):2031-5.

14. Phillips CB, Barrett JA, Losina E, et al. Incidence rates of dislocation, pulmonary embolism, and deep infection during the first six months after elective total hip replacement. J Bone Joint Surg Am. 2003;85-A:20.

15. Zenilman ME. MD. Delirium An Important Postoperative Complication. JAMA 2017 Jan 3;317(1):7778.

16. Chung KS, Lee JK, Park JS, Choi CH. Risk factors of delirium in patients undergoing total knee arthroplasty. Arch Gerontol Geriatr. 2015;60:443-7.

17. Scott JE, Mathias JL, Kneebone AC. Incidence of delirium following total joint replacement in older adults: a meta-analysis. Gen Hosp Psychiatry. 2015;37(3):223-9.

18. Robert K. Merrill1\& John M. Ibrahim, el at. Analysis and Review of Automated Risk Calculators Used to Predict Postoperative Complications After Orthopedic Surgery.Current Reviews in Musculoskeletal Medicine (2020) 13:298-308.

19. Monk TG, Saini V, Weldon BC, et al. Anesthetic management and one-year mortality after noncardiac surgery. Anesth Analg. 2005;100:4.

20. Higuera CA, Elsharkawy K, Klika AK, et al. 2010 Mid-America Orthopaedic Association Physician in Training Award: predictors of early adverse outcomes after knee and hip arthroplasty in geriatric patients. Clin Orthop Relat Res 2011; 469:1391. 21 Eymard F, Parsons C, Edwards MH, Petit-Dop F, Reginster JY, et al. Diabetes is a risk factor for knee osteoarthritis progression. Osteoarthritis Cartilage 2015;23: 851-9.

21. Saucedo JM, Marecek GS, Wanke TR, Lee J, Stulberg SD, Puri L. Understanding Readmission After Primary Total Hip and Knee Arthroplasty: Who's At Risk? Journal of Arthroplasty. 2014;29(2):256-60.

22. Illingworth KD, El Bitar YF, Banerjee D, Scaife SL, Saleh KJ. Inpatient mortality after primary total hip arthroplasty: analysis from the National Inpatient Sample database. J Arthroplasty. 2015;30:369-73.

23. Seltzer MH, Bastidas JA, Cooper DM, Engler P, Slocum B, Fletcher HS. Instant nutritional assessment. JPEN J Parenter Enteral Nutr. 1979;3:157-9.

24. Anmol Gupta MBA, et al. Serum albumin levels predict which patients are at increased risk for complications following surgical management of acute osteoporotic vertebral compression fractures. The Spine Journal. 2019;19:1796-802. 
25. de Mutsert R, Grootendorst D, Indemans F, Boeschoten E, Krediet R, Dekker F. Association between serum albumin and mortality in dialysis patients is partly explained by inflammation, and not by malnutrition. J Ren Nutr. 2009;19:127-35.

26. Mansmann U, Rieger A, Strahwald B, Crispin A. Risk calculators-methods, development, implementation, and validation. Int J Colorectal Dis. 2016;31(6):1111-6.

\section{Figures}

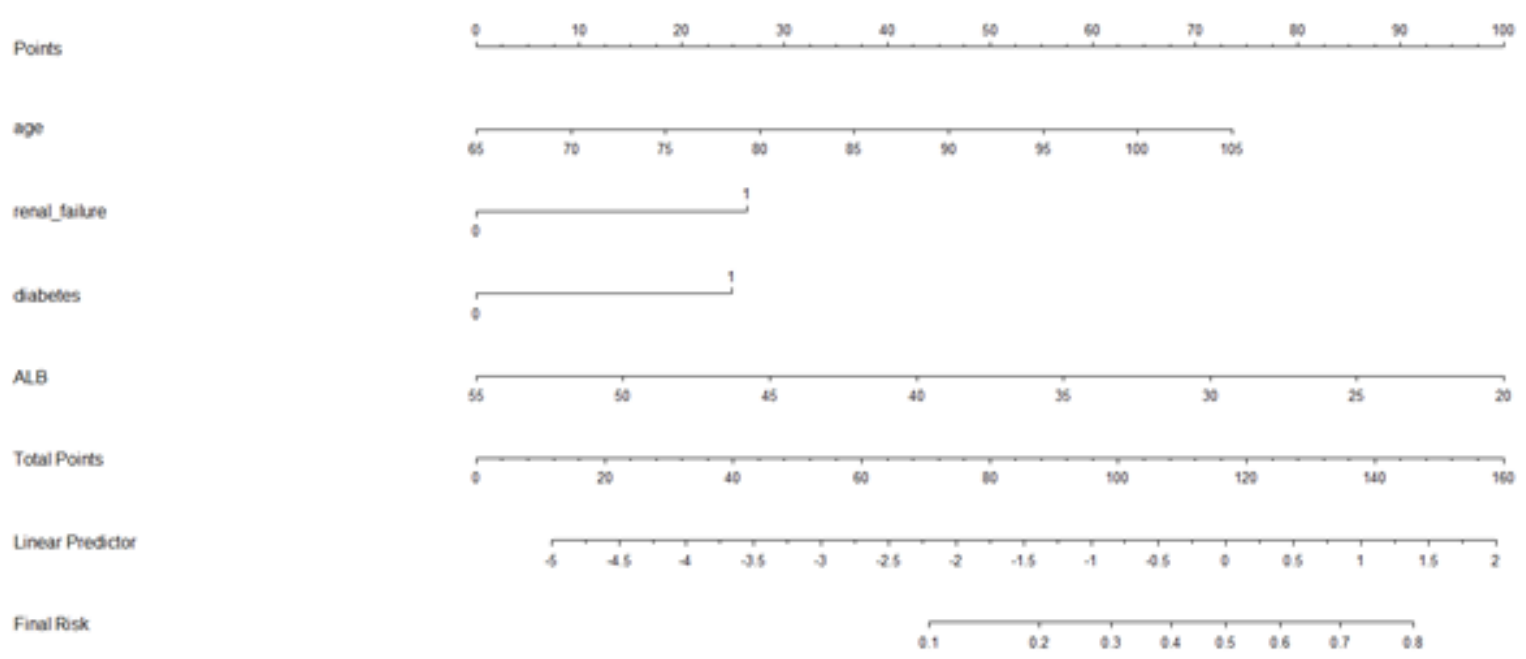

\section{Figure 1}

Nomogram. The Morbidity and Mortality Acute Predictor (arthro-MAP). The nomogram computes the probability of having a postoperative complication. In order to compute the predicted complication probability, a vertical line is to be drawn from the values of the individual variables to the scale for points on the top. Then a vertical line from the total points to the corresponding predicted complication probability. 


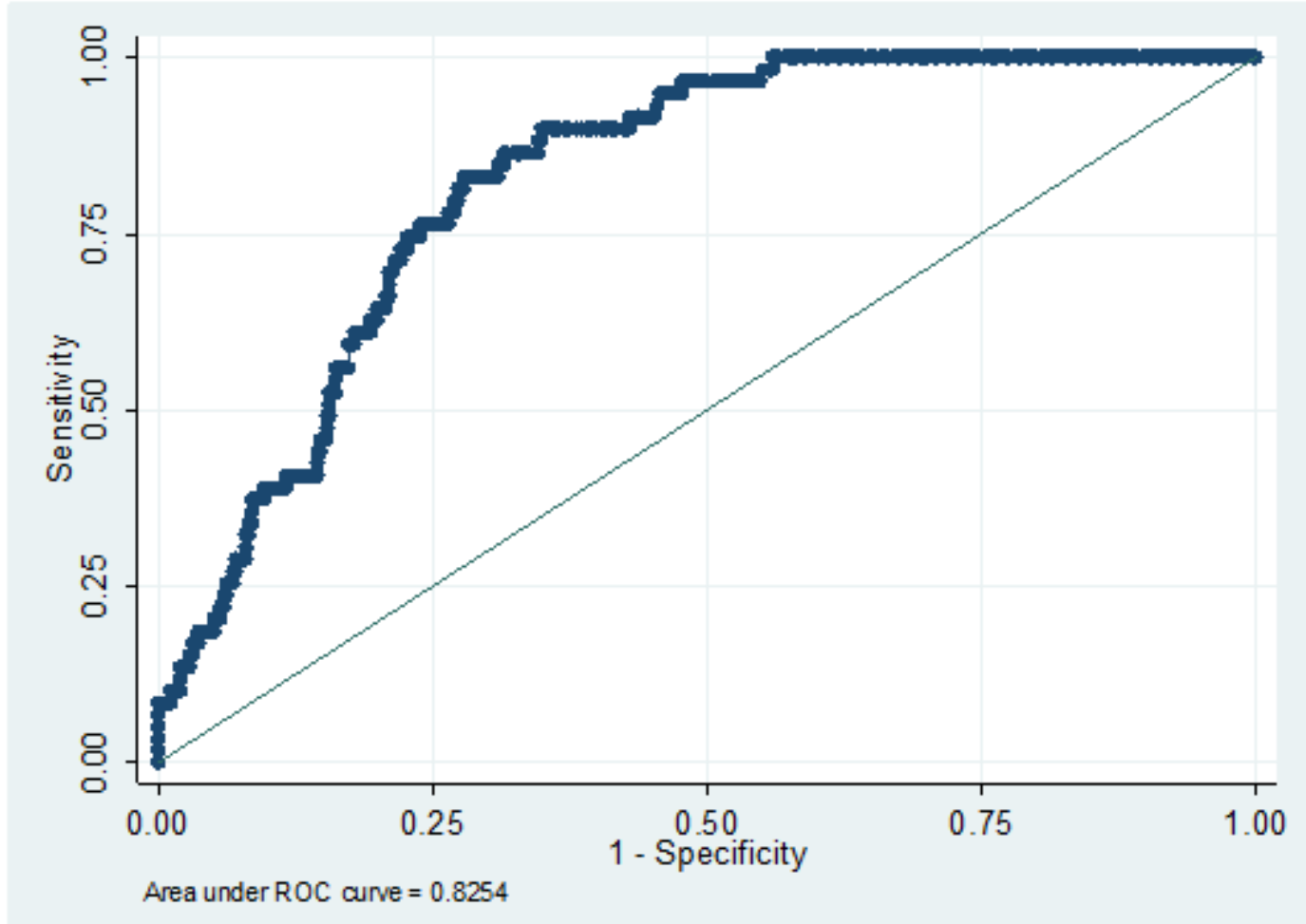

Figure 2

ROC curve. It also name sensitivity curve, the AUC (Area under curve) can evaluate the nomogram model discrimination degree. $A U C>0.6$ : may make sence; $A U C>0.7$ : Not bad; $A U C>0.8$ : Excellent.

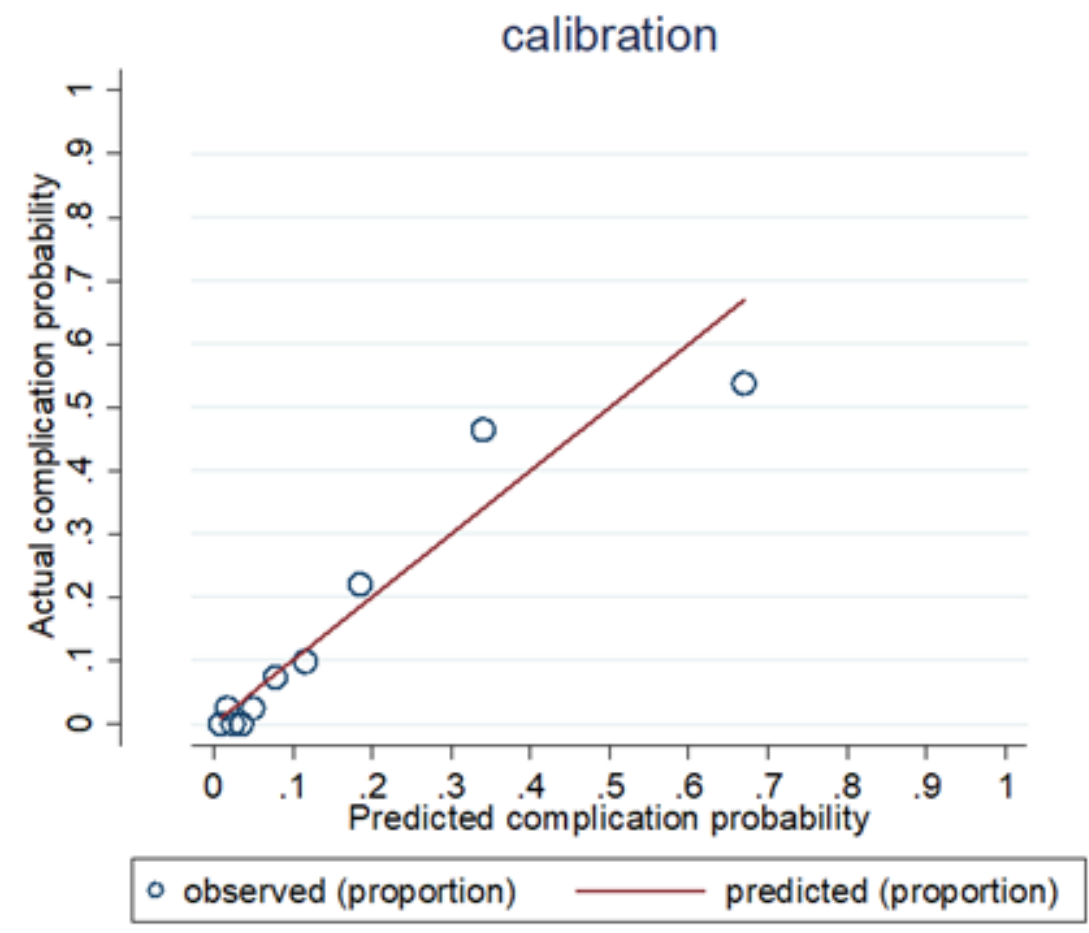

Figure 3 
Hosmer -Lemeshow test. The null hypothesis is that the fitting probability pi is grouped by 10 decile, and the difference between the fitted value and the observed value in each group, $p<0.05$ shows that the scatter separation is significantly deviated from the reference line, the predicted value is not equal to the actual value. Otherwise, the test passes, the predicted value is equal to the actual value.

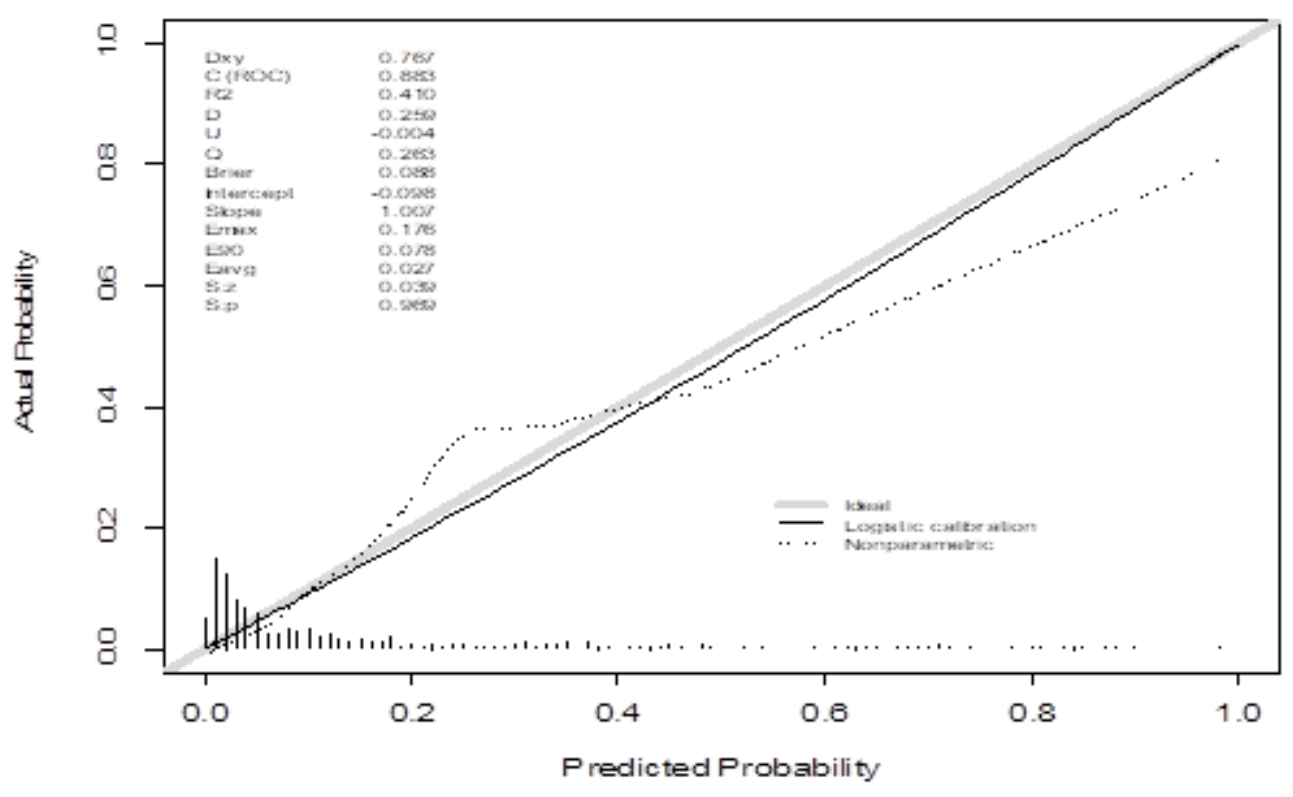

Figure 4

Calibration plot. A calibration plot compares the model's predicted probabilities and observed proportions. The diagonal line reflects the ideal situation (predicted probability = observed proportion). The curve represents the relation nonparametrically. The calibration curve is a straight line with a slope close to 1 , indicating that this model predicts postoperative complication risk in elderly total hip replacement patients consistent with the actual risk. 